\title{
WG-MOX Fuel Zr-Tube Neutron Spectrum Comparison in ATR and PWR
}

G. S. Chang

February 2005

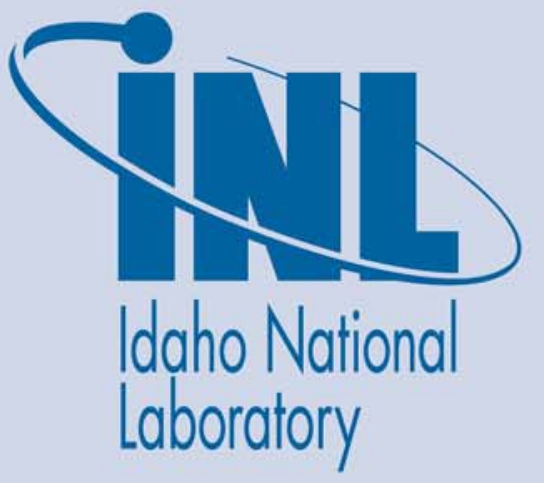

The INL is a U.S. Department of Energy National Laboratory operated by Battelle Energy Alliance 


\title{
WG-MOX Fuel Zr-tube Neutron Spectrum Comparison in ATR and PWR
}

\author{
G. S. Chang
}

February 2005

Idaho National Laboratory

Reactor and Nuclear Physics Department

Idaho Falls, Idaho 83415

Prepared for the

U.S. Department of Energy

Office of Fissile Material Disposition Program

Under DOE Idaho Operations Office

Contract DE-AC07-05ID14517 


\begin{abstract}
An experiment containing WG-MOX fuel has been designed and irradiated from 1998 to 2004 in the Advanced Test Reactor (ATR) at the Idaho National Engineering and Environmental Laboratory (INEEL). Important neutronics parameters were computed using novel Monte Carlo methods. The purpose of this summary is to compare the Weapons-Grade Mixed Oxide fuel (WG-MOX) Zr-tube's neutron spectrum in ATR and PWR. The results indicate that the Zrtube's neutron spectrum in ATR are softer than in PWR.
\end{abstract}




\section{CONTENTS}

ABSTRACT ..........................................................................................

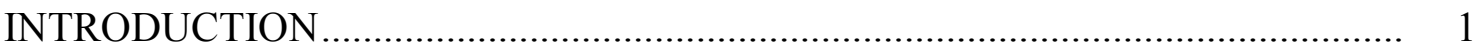

WG-MOX FUEL PIN MODEL IN ATR AND PWR LATTICE MODEL ................ 1

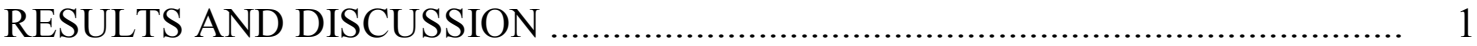

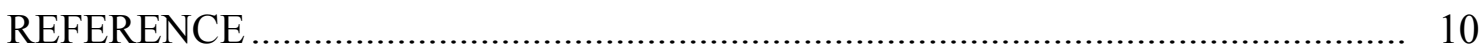

\section{FIGURES}

Figure 1. ATR MCNP core model cross-section view........................................ 2

Figure 2. Detailed radial cross-sectional view of the WG-MOX fuel test assembly.. 3

Figure 3. WG-MOX fuel pin spectrum comparison in ATR small I-irradiation

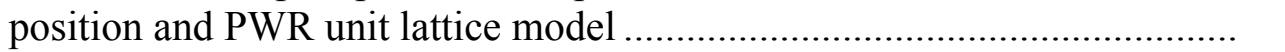

Figure 4. Comparison of the WG-MOX Zr-tube's neutron spectrum in ATR and PWR

\section{TABLE}

Table 1. MCNP-calculated WG-MOX Zr-tube's neutron spectrum (relative neutron flux fraction per lethargy) in ATR and PWR 


\section{WG-MOX FUEL ZR-TUBE NEUTRON SPECTRUMCOMPARISON IN ATR AND PWR}

\section{INTRODUCTION}

In support of potential licensing of mixed oxide (MOX) fuel made from weapons-grade (WG) plutonium and depleted uranium for use in U. S. reactors, an experiment containing WG-MOX fuel has been designed and irradiated from 1998 to 2004 in the Advanced Test Reactor (ATR) at the Idaho National Engineering and Environmental Laboratory (INEEL). A simple, uninstrumented, test assembly containing nine MOX fuel capsules with neutron monitor wires was inserted into the ATR reflector. Important neutronics parameters were computed using novel Monte Carlo methods. The purpose of this summary is to compare the Weapons-Grade Mixed Oxide fuel (WG-MOX) Zr-tube's neutron spectrum in ATR and PWR.

\section{WG-MOX FUEL PIN MODEL IN ATR AND PWR LATTICE MODEL}

The thermal neutron flux in the ATR reflector is dependent on the outer shim cylinder positions, the neck shim positions, and the depletion of the fuel and Boron-10 in the nearest lobe. The plan view of the small I-irradiation positions in ATR is shown in Figure 1. There are 3 MOX fuel test sections axially, with the center section at the core midplane, and 3 fuel capsules (SS-304L) in each section, for a total of 9 fuel capsules in the test assembly. Each capsule contains one fuel pin. Each fuel pin contains $15 \mathrm{MOX}$ fuel pellets and each fuel pellet stack is $0.415 \mathrm{~cm}$ in radius and $15.24 \mathrm{~cm}$ in length. The fuel pellets are enclosed in $0.0686 \mathrm{~cm}$ thick Zircaloy cladding tubes. To meet the ATR safety requirement, then, Zircaloy cladding tube was enclosed in $0.1016 \mathrm{~cm}$ thick stainless-steel (SST) tube. A gap of $0.0025 \mathrm{~cm}$ separates the WG-MOX pellet from the Zircaloy cladding. The fill gas for the fuel pins and capsules was helium at atmospheric pressure. The radial view of the test assembly and capsule is shown in Figure 2. The WG-MOX fuel pellet contains 5.0 wt $\% \mathrm{PuO}_{2}$ with ${ }^{239-242} \mathrm{Pu}$ represented with 93.6, 5.9, 0.4, and $0.1 \mathrm{wt} \% \mathrm{Pu}$, respectively.

A typical PWR unit cell has been chosen as the basis for the fuel neutronics burnup characteristics comparison with the WG-MOX fuel irradiated in ATR. A typical PWR $17 \times 17$ fuel-element array lattice with fuel pin-to-pin pitch of $1.43 \mathrm{~cm}$ was chosen as a reference case in this study.

\section{RESULTS AND DISCUSSION}

The comparison of the ATR with WG-MOX fuel at Beginning of Life (BOL) and End of Life (EOL), and the PWR unit lattice neutron flux spectra in the fuel pin is shown in Figure 3, which shows that the neutron spectrum in the ATR test position is somewhat softer than the neutron spectrum in a PWR. The MCNP ${ }^{1}$-calculated WG-MOX fuel pin and Zr-tube's neutron spectrum in ATR with WG-MOX fuel at BOL and EOL, and PWR are plotted in Fig. 4, which also shows a softer neutron flux spectrum in ATR. Note that the major source of fast neutron of WG-MOX fuel capsule in ATR is coming from the WG-MOX fuel pin alone. Toward the end of WG-MOX irradiation life, the $\mathrm{Pu}-239$ depleted about 92 atom\%, which makes the $\mathrm{Zr}$ 's tube neutron flux spectrum softer than the spectrum at the beginning of irradiation life. In addition, there is a significant portion of the fast neutron flux is coming from the adjoining pins in PWR, which make the Zr's tube fast neutron fluence rather higher than Zr's tube fast neutron fluence in ATR for the same MOX fuel burnup. The MCNP-calculated detailed results are also tabulated in Table 1. 


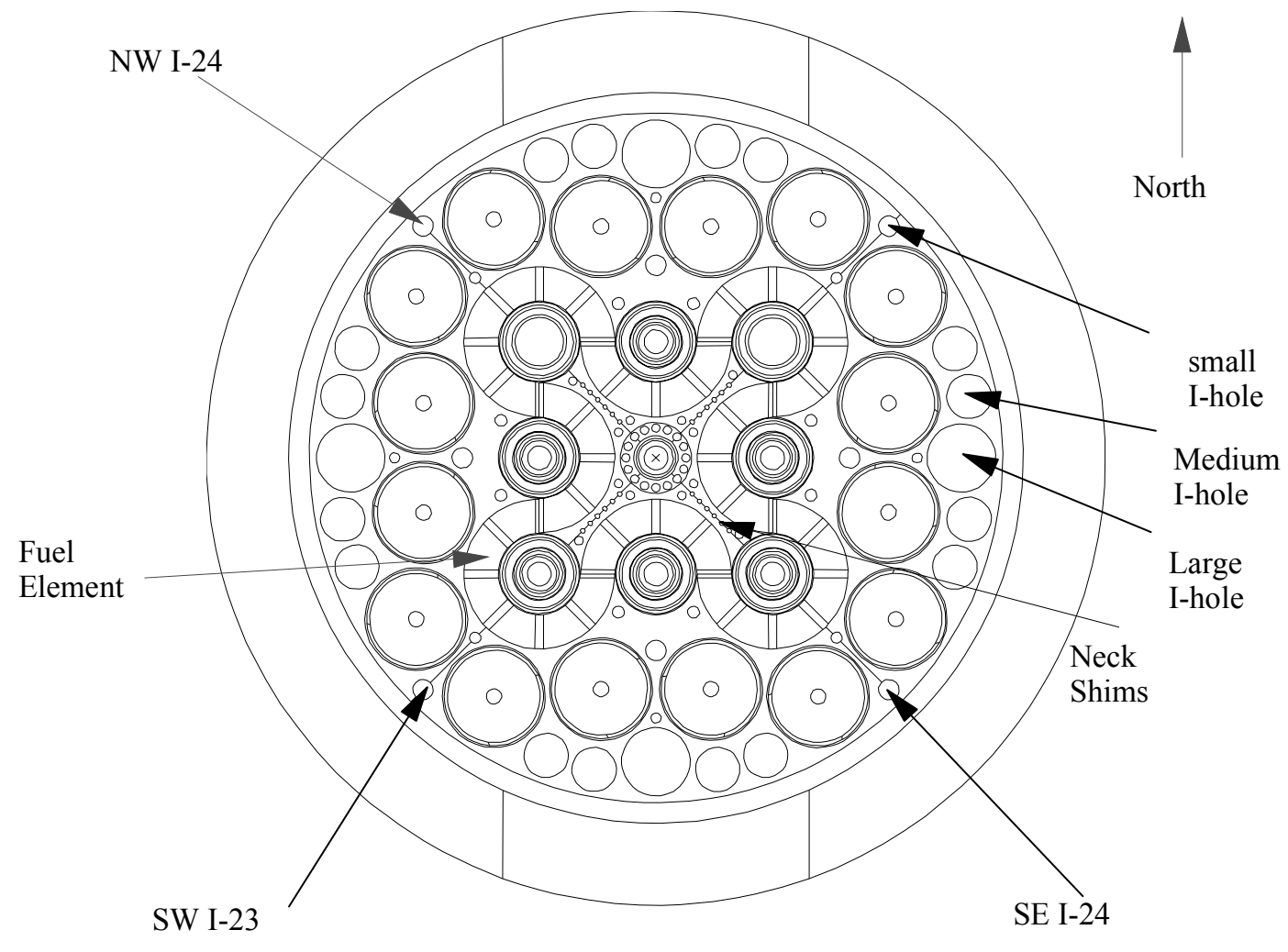

Figure 1. ATR MCNP core model cross-section view. 


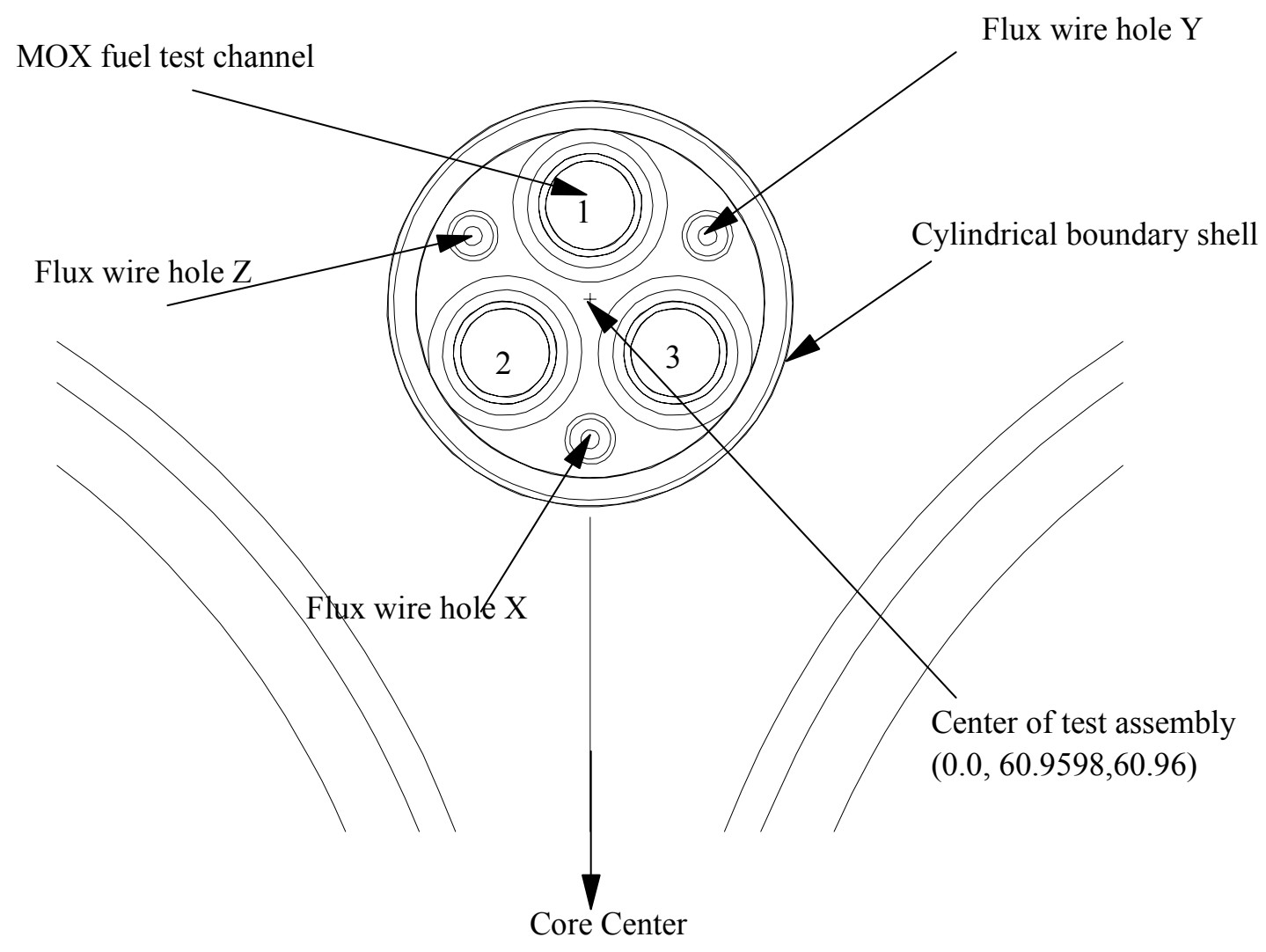

Figure 2. Detailed radial cross-sectional view of the WG-MOX fuel test assembly 


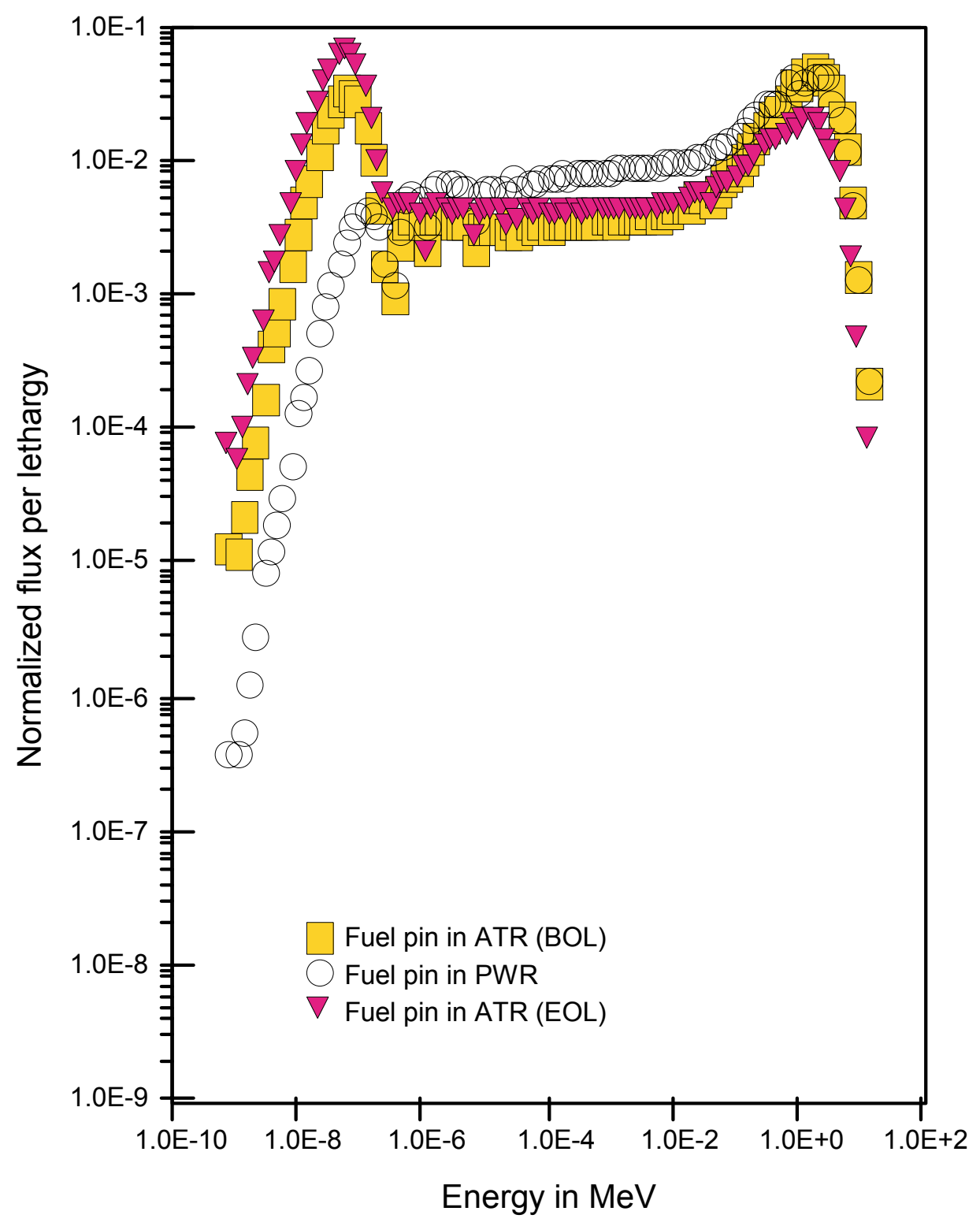

Figure 3. WG-MOX fuel pin spectrum comparison in ATR small I-irradiation position and PWR unit lattice model. 


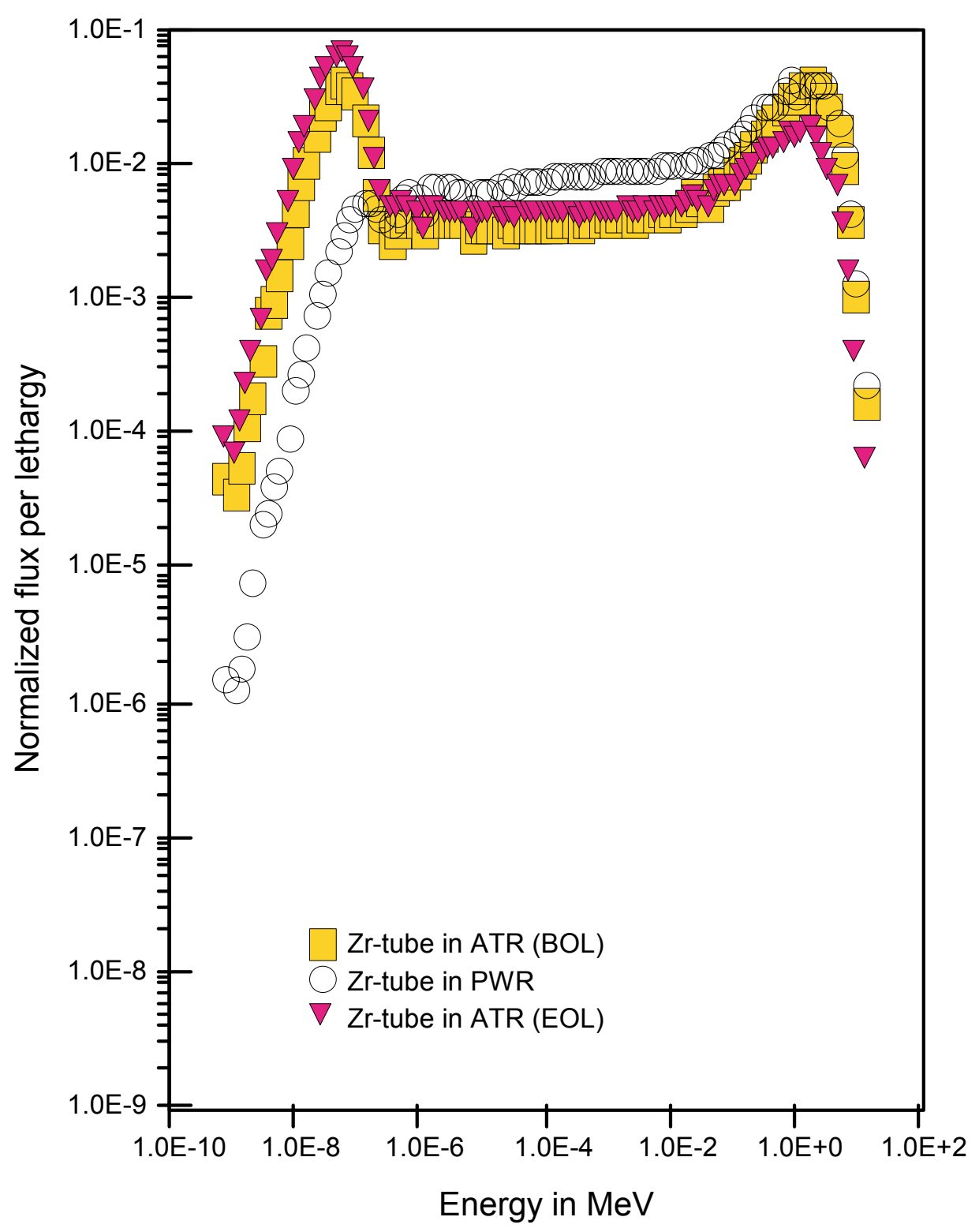

Figure 4. Comparison of the WG-MOX Zr-tube's neutron spectrum in ATR and PWR 
Table 1. MCNP-calculated WG-MOX Zr-tube's neutron spectrum (relative neutron flux fraction per lethargy) in ATR and PWR

$\underline{\text { ATR WG-MOX at BOL ATR WG-MOX at EOL }}$

Energy MeV

Fuel pin

Fuel pin
1.404E-005

5.154E-005

1.119E-009

$1.377 \mathrm{E}-005$

1.437E-009

2.393E-005

$1.845 \mathrm{E}-009$

2.369E-009

3.041E-009

3.905E-009

5.014E-009

6.439E-009

8.267E-009

$1.062 \mathrm{E}-008$

1.363E-008

1.750E-008

2.247E-008

$2.886 \mathrm{E}-008$

3.705E-008

4.758E-008

6.109E-008
4.756E-004

$9.160 \mathrm{E}-004$

5.293E-005

9.205E-005

$1.848 \mathrm{E}-004$

$5.941 \mathrm{E}-00$

1.001E-003

1.797E-003

3.335E-003

5.632E-003

8.195E-003

1.297E-002

$1.975 \mathrm{E}-002$

(1)

$2.558 \mathrm{E}-002$

3.293E-002

4.183E-002

3.310E-002

3.727E-002

$1.065 \mathrm{E}-003$

1.703E-003

$2.896 \mathrm{E}-003$

8.276E-003

4.639E-002
5.133E-003

$1.163 \mathrm{E}-002$

2.626E-002
1.772E-002

8.387E-005

6.423E-005

1.081E-004

$2.158 \mathrm{E}-004$

$2.502 \mathrm{E}-004$

4.123E-004

3.713E-004

6.693E-004

7.454E-004

$9.438 \mathrm{E}-006$

2.299E-005

1.509E-003

$1.676 \mathrm{E}-003$

1.283E-005

$2.824 \mathrm{E}-005$

$1.856 \mathrm{E}-003$

$2.013 \mathrm{E}-003$

$2.065 \mathrm{E}-005$

4.254E-005

2.996E-003

3.216E-003

3.202E-005

$5.826 \mathrm{E}-005$

5.003E-003

5.345E-003

$5.875 \mathrm{E}-005$

1.002E-004

8.785E-003

$9.310 \mathrm{E}-003$

$1.396 \mathrm{E}-004$

2.334E-004

$1.395 \mathrm{E}-002$

1.471E-002

$1.934 \mathrm{E}-004$

3.037E-004

1.933E-002

$2.026 \mathrm{E}-002$

3.066E-004

4.542E-004

$2.916 \mathrm{E}-002$

3.043E-002

$5.469 \mathrm{E}-004$

7.819E-004

4.233E-002

4.407E-002

8.575E-00

$1.186 \mathrm{E}-003$

5.410E-002

1.300E-003

1.739E-003

6.512E-002

6.720E-002

1.869E-003

$2.450 \mathrm{E}-003$

7.093E-002

7.300E-002

2.575E-003 3.304E-003 


\begin{tabular}{|c|c|c|c|c|c|c|}
\hline 7.844E-008 & $3.525 \mathrm{E}-002$ & $4.326 \mathrm{E}-002$ & $6.542 \mathrm{E}-002$ & $6.708 \mathrm{E}-002$ & $3.388 \mathrm{E}-003$ & 4.290E-003 \\
\hline $1.007 \mathrm{E}-007$ & $3.062 \mathrm{E}-002$ & $3.745 \mathrm{E}-002$ & $5.616 \mathrm{E}-002$ & $5.764 \mathrm{E}-002$ & 4.165E-003 & $5.245 \mathrm{E}-003$ \\
\hline $1.293 \mathrm{E}-007$ & $2.001 \mathrm{E}-002$ & $2.460 \mathrm{E}-002$ & $3.702 \mathrm{E}-002$ & $3.794 \mathrm{E}-002$ & 4.564E-003 & $5.792 \mathrm{E}-003$ \\
\hline $1.661 \mathrm{E}-007$ & $1.135 \mathrm{E}-002$ & $1.438 \mathrm{E}-002$ & $2.206 \mathrm{E}-002$ & $2.264 \mathrm{E}-002$ & $4.342 \mathrm{E}-003$ & $5.715 \mathrm{E}-003$ \\
\hline 2.132E-007 & 4.987E-003 & 6.993E-003 & $1.108 \mathrm{E}-002$ & $1.150 \mathrm{E}-002$ & $3.416 \mathrm{E}-003$ & $5.120 \mathrm{E}-003$ \\
\hline $2.738 \mathrm{E}-007$ & $1.838 \mathrm{E}-003$ & $3.699 \mathrm{E}-003$ & $6.122 \mathrm{E}-003$ & $6.559 \mathrm{E}-003$ & $1.811 \mathrm{E}-003$ & 4.084E-003 \\
\hline $3.515 \mathrm{E}-007$ & $1.098 \mathrm{E}-003$ & $2.920 \mathrm{E}-003$ & $4.836 \mathrm{E}-003$ & $5.209 \mathrm{E}-003$ & $1.280 \mathrm{E}-003$ & $3.761 \mathrm{E}-003$ \\
\hline 4.514E-007 & $2.665 \mathrm{E}-003$ & $3.564 \mathrm{E}-003$ & $5.180 \mathrm{E}-003$ & $5.306 \mathrm{E}-003$ & $3.352 \mathrm{E}-003$ & 4.662E-003 \\
\hline $5.796 \mathrm{E}-007$ & $3.943 \mathrm{E}-003$ & 4.308E-003 & 5.323E-003 & 5.337E-003 & $5.676 \mathrm{E}-003$ & $6.210 \mathrm{E}-003$ \\
\hline $7.442 \mathrm{E}-007$ & $4.096 \mathrm{E}-003$ & 4.437E-003 & 4.983E-003 & $5.132 \mathrm{E}-003$ & $6.229 \mathrm{E}-003$ & $6.520 \mathrm{E}-003$ \\
\hline $9.556 \mathrm{E}-007$ & $3.813 \mathrm{E}-003$ & 4.132E-003 & 4.247E-003 & $4.508 \mathrm{E}-003$ & $5.813 \mathrm{E}-003$ & $6.171 \mathrm{E}-003$ \\
\hline $1.227 \mathrm{E}-006$ & $2.485 \mathrm{E}-003$ & $3.399 \mathrm{E}-003$ & $2.140 \mathrm{E}-003$ & $3.462 \mathrm{E}-003$ & $3.410 \mathrm{E}-003$ & 4.819E-003 \\
\hline $1.576 \mathrm{E}-006$ & $4.218 \mathrm{E}-003$ & 4.448E-003 & $4.768 \mathrm{E}-003$ & 4.903E-003 & $6.947 \mathrm{E}-003$ & $7.057 \mathrm{E}-003$ \\
\hline $2.023 \mathrm{E}-006$ & 4.195E-003 & 4.373E-003 & $4.865 \mathrm{E}-003$ & $4.910 \mathrm{E}-003$ & 7.117E-003 & 7.154E-003 \\
\hline $2.598 \mathrm{E}-006$ & $4.215 \mathrm{E}-003$ & 4.367E-003 & 4.749E-003 & 4.782E-003 & $7.096 \mathrm{E}-003$ & $7.116 \mathrm{E}-003$ \\
\hline $3.335 \mathrm{E}-006$ & $4.027 \mathrm{E}-003$ & 4.181E-003 & 4.253E-003 & $4.468 \mathrm{E}-003$ & $7.038 \mathrm{E}-003$ & $7.058 \mathrm{E}-003$ \\
\hline 4.283E-006 & $3.989 \mathrm{E}-003$ & 4.139E-003 & 4.639E-003 & 4.647E-003 & $6.970 \mathrm{E}-003$ & $6.977 \mathrm{E}-003$ \\
\hline $5.499 \mathrm{E}-006$ & $3.857 \mathrm{E}-003$ & 4.089E-003 & $4.475 \mathrm{E}-003$ & 4.604E-003 & $6.544 \mathrm{E}-003$ & 6.642E-003 \\
\hline $7.061 \mathrm{E}-006$ & $2.517 \mathrm{E}-003$ & $3.277 \mathrm{E}-003$ & $2.806 \mathrm{E}-003$ & $3.656 \mathrm{E}-003$ & $4.042 \mathrm{E}-003$ & $5.171 \mathrm{E}-003$ \\
\hline $9.066 \mathrm{E}-006$ & $3.550 \mathrm{E}-003$ & $3.864 \mathrm{E}-003$ & $4.414 \mathrm{E}-003$ & $4.540 \mathrm{E}-003$ & 6.192E-003 & $6.558 \mathrm{E}-003$ \\
\hline $1.164 \mathrm{E}-005$ & $3.574 \mathrm{E}-003$ & $3.864 \mathrm{E}-003$ & 4.619E-003 & $4.662 \mathrm{E}-003$ & $6.455 \mathrm{E}-003$ & $6.748 \mathrm{E}-003$ \\
\hline $1.495 \mathrm{E}-005$ & $3.473 \mathrm{E}-003$ & $3.754 \mathrm{E}-003$ & $4.481 \mathrm{E}-003$ & $4.507 \mathrm{E}-003$ & $6.465 \mathrm{E}-003$ & $6.825 \mathrm{E}-003$ \\
\hline 1.919E-005 & $3.660 \mathrm{E}-003$ & $3.899 \mathrm{E}-003$ & $4.440 \mathrm{E}-003$ & $4.501 \mathrm{E}-003$ & $6.879 \mathrm{E}-003$ & $7.120 \mathrm{E}-003$ \\
\hline
\end{tabular}




\begin{tabular}{|c|c|c|c|c|c|c|}
\hline $2.465 \mathrm{E}-005$ & $3.123 \mathrm{E}-003$ & $3.651 \mathrm{E}-003$ & $3.660 \mathrm{E}-003$ & 4.147E-003 & 5.894E-003 & $6.720 \mathrm{E}-003$ \\
\hline $3.165 \mathrm{E}-005$ & $3.916 \mathrm{E}-003$ & $4.090 \mathrm{E}-003$ & $4.591 \mathrm{E}-003$ & 4.610E-003 & 7.811E-003 & 7.877E-003 \\
\hline $4.063 \mathrm{E}-005$ & $3.311 \mathrm{E}-003$ & $3.800 \mathrm{E}-003$ & $3.799 \mathrm{E}-003$ & 4.244E-003 & $6.563 \mathrm{E}-003$ & $7.269 \mathrm{E}-003$ \\
\hline $5.217 \mathrm{E}-005$ & $3.738 \mathrm{E}-003$ & $3.934 \mathrm{E}-003$ & $4.518 \mathrm{E}-003$ & $4.546 \mathrm{E}-003$ & 7.648E-003 & $7.864 \mathrm{E}-003$ \\
\hline $6.699 \mathrm{E}-005$ & $3.538 \mathrm{E}-003$ & $3.914 \mathrm{E}-003$ & $4.276 \mathrm{E}-003$ & $4.495 \mathrm{E}-003$ & $7.255 \mathrm{E}-003$ & 7.727E-003 \\
\hline 8.602E-005 & $3.726 \mathrm{E}-003$ & $3.993 \mathrm{E}-003$ & 4.443E-003 & $4.551 \mathrm{E}-003$ & 7.759E-003 & $8.040 \mathrm{E}-003$ \\
\hline $1.105 \mathrm{E}-004$ & $3.643 \mathrm{E}-003$ & $3.979 \mathrm{E}-003$ & $4.252 \mathrm{E}-003$ & $4.500 \mathrm{E}-003$ & 7.851E-003 & $8.242 \mathrm{E}-003$ \\
\hline $1.418 \mathrm{E}-004$ & 3.791E-003 & $4.021 \mathrm{E}-003$ & 4.394E-003 & 4.498E-003 & 8.331E-003 & $8.536 \mathrm{E}-003$ \\
\hline $1.821 \mathrm{E}-004$ & $3.916 \mathrm{E}-003$ & $4.119 \mathrm{E}-003$ & $4.539 \mathrm{E}-003$ & $4.590 \mathrm{E}-003$ & $8.516 \mathrm{E}-003$ & $8.646 \mathrm{E}-003$ \\
\hline $2.338 \mathrm{E}-004$ & $3.823 \mathrm{E}-003$ & 4.144E-003 & 4.394E-003 & $4.615 \mathrm{E}-003$ & $8.380 \mathrm{E}-003$ & 8.704E-003 \\
\hline $3.002 \mathrm{E}-004$ & $4.029 \mathrm{E}-003$ & $4.358 \mathrm{E}-003$ & $4.643 \mathrm{E}-003$ & $4.846 \mathrm{E}-003$ & $8.500 \mathrm{E}-003$ & $8.768 \mathrm{E}-003$ \\
\hline $3.855 \mathrm{E}-004$ & $3.734 \mathrm{E}-003$ & $3.901 \mathrm{E}-003$ & $4.258 \mathrm{E}-003$ & $4.295 \mathrm{E}-003$ & 8.888E-003 & 8.947E-003 \\
\hline $4.950 \mathrm{E}-004$ & $3.970 \mathrm{E}-003$ & 4.157E-003 & 4.547E-003 & $4.572 \mathrm{E}-003$ & $8.970 \mathrm{E}-003$ & $9.045 \mathrm{E}-003$ \\
\hline $6.356 \mathrm{E}-004$ & $4.028 \mathrm{E}-003$ & $4.275 \mathrm{E}-003$ & $4.583 \mathrm{E}-003$ & $4.668 \mathrm{E}-003$ & 8.957E-003 & $9.125 \mathrm{E}-003$ \\
\hline 8.161E-004 & 4.087E-003 & 4.327E-003 & 4.639E-003 & 4.714E-003 & $9.206 \mathrm{E}-003$ & $9.282 \mathrm{E}-003$ \\
\hline $1.048 \mathrm{E}-003$ & $4.158 \mathrm{E}-003$ & $4.437 \mathrm{E}-003$ & 4.689E-003 & $4.845 \mathrm{E}-003$ & $9.184 \mathrm{E}-003$ & $9.354 \mathrm{E}-003$ \\
\hline $1.346 \mathrm{E}-003$ & $4.042 \mathrm{E}-003$ & $4.256 \mathrm{E}-003$ & $4.524 \mathrm{E}-003$ & $4.603 \mathrm{E}-003$ & $9.246 \mathrm{E}-003$ & $9.369 \mathrm{E}-003$ \\
\hline $1.728 \mathrm{E}-003$ & $4.225 \mathrm{E}-003$ & 4.479E-003 & 4.711E-003 & $4.826 \mathrm{E}-003$ & $9.410 \mathrm{E}-003$ & $9.506 \mathrm{E}-003$ \\
\hline 2.219E-003 & 4.317E-003 & 4.634E-003 & 4.801E-003 & $4.956 \mathrm{E}-003$ & $9.539 \mathrm{E}-003$ & $9.631 \mathrm{E}-003$ \\
\hline 2.849E-003 & 4.093E-003 & $4.375 \mathrm{E}-003$ & 4.549E-003 & 4.674E-003 & $9.490 \mathrm{E}-003$ & $9.608 \mathrm{E}-003$ \\
\hline $3.658 \mathrm{E}-003$ & 4.339E-003 & $4.529 \mathrm{E}-003$ & $4.785 \mathrm{E}-003$ & 4.841E-003 & $9.675 \mathrm{E}-003$ & $9.715 \mathrm{E}-003$ \\
\hline 4.697E-003 & $4.434 \mathrm{E}-003$ & $4.660 \mathrm{E}-003$ & $4.815 \mathrm{E}-003$ & 4.901E-003 & $9.871 \mathrm{E}-003$ & $9.965 \mathrm{E}-003$ \\
\hline $6.030 \mathrm{E}-003$ & $4.423 \mathrm{E}-003$ & $4.605 \mathrm{E}-003$ & $4.786 \mathrm{E}-003$ & $4.826 \mathrm{E}-003$ & 9.859E-003 & $9.854 \mathrm{E}-003$ \\
\hline
\end{tabular}




\begin{tabular}{|c|c|c|c|c|c|c|}
\hline 7.743E-003 & 4.707E-003 & 4.944E-003 & $5.027 \mathrm{E}-003$ & $5.143 \mathrm{E}-003$ & 1.012E-002 & $1.013 \mathrm{E}-002$ \\
\hline $9.942 \mathrm{E}-003$ & 4.604E-003 & $4.756 \mathrm{E}-003$ & $4.925 \mathrm{E}-003$ & $4.898 \mathrm{E}-003$ & $1.020 \mathrm{E}-002$ & $1.021 \mathrm{E}-002$ \\
\hline $1.277 \mathrm{E}-002$ & 4.932E-003 & $5.132 \mathrm{E}-003$ & $5.189 \mathrm{E}-003$ & $5.225 \mathrm{E}-003$ & $1.049 \mathrm{E}-002$ & $1.044 \mathrm{E}-002$ \\
\hline $1.639 \mathrm{E}-002$ & $5.062 \mathrm{E}-003$ & $5.213 \mathrm{E}-003$ & $5.268 \mathrm{E}-003$ & $5.241 \mathrm{E}-003$ & $1.072 \mathrm{E}-002$ & $1.066 \mathrm{E}-002$ \\
\hline $2.105 \mathrm{E}-002$ & $5.285 \mathrm{E}-003$ & $5.398 \mathrm{E}-003$ & 5.392E-003 & $5.372 \mathrm{E}-003$ & $1.104 \mathrm{E}-002$ & $1.098 \mathrm{E}-002$ \\
\hline 2.703E-002 & $6.214 \mathrm{E}-003$ & $6.506 \mathrm{E}-003$ & $6.254 \mathrm{E}-003$ & $6.352 \mathrm{E}-003$ & $1.159 \mathrm{E}-002$ & $1.149 \mathrm{E}-002$ \\
\hline $3.470 \mathrm{E}-002$ & $6.030 \mathrm{E}-003$ & $6.123 \mathrm{E}-003$ & $5.842 \mathrm{E}-003$ & $5.770 \mathrm{E}-003$ & 1.179E-002 & $1.168 \mathrm{E}-002$ \\
\hline 4.456E-002 & $5.550 \mathrm{E}-003$ & $5.558 \mathrm{E}-003$ & $5.236 \mathrm{E}-003$ & $5.146 \mathrm{E}-003$ & $1.258 \mathrm{E}-002$ & $1.244 \mathrm{E}-002$ \\
\hline $5.722 \mathrm{E}-002$ & $6.963 \mathrm{E}-003$ & 7.037E-003 & $6.462 \mathrm{E}-003$ & $6.450 \mathrm{E}-003$ & $1.334 \mathrm{E}-002$ & $1.317 \mathrm{E}-002$ \\
\hline 7.347E-002 & 8.306E-003 & 8.377E-003 & $7.450 \mathrm{E}-003$ & 7.432E-003 & $1.432 \mathrm{E}-002$ & $1.410 \mathrm{E}-002$ \\
\hline $9.433 \mathrm{E}-002$ & $8.520 \mathrm{E}-003$ & $8.411 \mathrm{E}-003$ & 7.098E-003 & $7.037 \mathrm{E}-003$ & $1.549 \mathrm{E}-002$ & $1.523 \mathrm{E}-002$ \\
\hline $1.211 \mathrm{E}-001$ & $9.727 \mathrm{E}-003$ & 9.419E-003 & 7.870E-003 & $7.681 \mathrm{E}-003$ & 1.709E-002 & $1.669 \mathrm{E}-002$ \\
\hline $1.555 \mathrm{E}-001$ & $1.203 \mathrm{E}-002$ & $1.168 \mathrm{E}-002$ & $9.317 \mathrm{E}-003$ & $9.162 \mathrm{E}-003$ & $1.906 \mathrm{E}-002$ & $1.857 \mathrm{E}-002$ \\
\hline $1.997 \mathrm{E}-001$ & $1.364 \mathrm{E}-002$ & $1.289 \mathrm{E}-002$ & $9.940 \mathrm{E}-003$ & $9.643 \mathrm{E}-003$ & $2.155 \mathrm{E}-002$ & $2.088 \mathrm{E}-002$ \\
\hline $2.564 \mathrm{E}-001$ & $1.643 \mathrm{E}-002$ & $1.513 \mathrm{E}-002$ & $1.126 \mathrm{E}-002$ & $1.077 \mathrm{E}-002$ & $2.477 \mathrm{E}-002$ & $2.388 \mathrm{E}-002$ \\
\hline $3.293 \mathrm{E}-001$ & $2.086 \mathrm{E}-002$ & $1.895 \mathrm{E}-002$ & $1.352 \mathrm{E}-002$ & $1.279 \mathrm{E}-002$ & $2.916 \mathrm{E}-002$ & $2.794 \mathrm{E}-002$ \\
\hline $4.228 \mathrm{E}-001$ & $2.498 \mathrm{E}-002$ & $2.241 \mathrm{E}-002$ & $1.507 \mathrm{E}-002$ & $1.421 \mathrm{E}-002$ & 2.889E-002 & $2.780 \mathrm{E}-002$ \\
\hline $5.428 \mathrm{E}-001$ & $2.693 \mathrm{E}-002$ & 2.389E-002 & $1.490 \mathrm{E}-002$ & $1.407 \mathrm{E}-002$ & 2.993E-002 & $2.883 \mathrm{E}-002$ \\
\hline $6.970 \mathrm{E}-001$ & $3.300 \mathrm{E}-002$ & $2.814 \mathrm{E}-002$ & $1.638 \mathrm{E}-002$ & $1.491 \mathrm{E}-002$ & 4.174E-002 & $3.977 \mathrm{E}-002$ \\
\hline $8.950 \mathrm{E}-001$ & 4.211E-002 & $3.542 \mathrm{E}-002$ & $1.993 \mathrm{E}-002$ & $1.786 \mathrm{E}-002$ & 4.780E-002 & $4.545 \mathrm{E}-002$ \\
\hline $1.149 \mathrm{E}+000$ & 4.307E-002 & $3.577 \mathrm{E}-002$ & $1.904 \mathrm{E}-002$ & $1.707 \mathrm{E}-002$ & $3.594 \mathrm{E}-002$ & $3.425 \mathrm{E}-002$ \\
\hline $1.476 \mathrm{E}+000$ & $5.044 \mathrm{E}-002$ & $4.131 \mathrm{E}-002$ & $2.144 \mathrm{E}-002$ & $1.901 \mathrm{E}-002$ & $4.355 \mathrm{E}-002$ & 4.149E-002 \\
\hline $1.895 \mathrm{E}+000$ & $5.497 \mathrm{E}-002$ & $4.482 \mathrm{E}-002$ & $2.277 \mathrm{E}-002$ & $2.010 \mathrm{E}-002$ & 4.386E-002 & $4.169 \mathrm{E}-002$ \\
\hline
\end{tabular}




$\begin{array}{ccccccc}2.433 \mathrm{E}+000 & 5.209 \mathrm{E}-002 & 4.140 \mathrm{E}-002 & 1.950 \mathrm{E}-002 & 1.668 \mathrm{E}-002 & 4.475 \mathrm{E}-002 & 4.256 \mathrm{E}-002 \\ 3.124 \mathrm{E}+000 & 4.704 \mathrm{E}-002 & 3.646 \mathrm{E}-002 & 1.577 \mathrm{E}-002 & 1.303 \mathrm{E}-002 & 4.465 \mathrm{E}-002 & 4.245 \mathrm{E}-002 \\ 4.011 \mathrm{E}+000 & 3.650 \mathrm{E}-002 & 2.798 \mathrm{E}-002 & 1.225 \mathrm{E}-002 & 1.003 \mathrm{E}-002 & 2.936 \mathrm{E}-002 & 2.772 \mathrm{E}-002 \\ 5.150 \mathrm{E}+000 & 2.518 \mathrm{E}-002 & 1.922 \mathrm{E}-002 & 8.609 \mathrm{E}-003 & 7.112 \mathrm{E}-003 & 2.236 \mathrm{E}-002 & 2.120 \mathrm{E}-002 \\ 6.613 \mathrm{E}+000 & 1.360 \mathrm{E}-002 & 1.040 \mathrm{E}-002 & 4.675 \mathrm{E}-003 & 3.807 \mathrm{E}-003 & 1.316 \mathrm{E}-002 & 1.249 \mathrm{E}-002 \\ 8.491 \mathrm{E}+000 & 5.537 \mathrm{E}-003 & 4.241 \mathrm{E}-003 & 1.979 \mathrm{E}-003 & 1.624 \mathrm{E}-003 & 5.097 \mathrm{E}-003 & 4.848 \mathrm{E}-003 \\ 1.090 \mathrm{E}+001 & 1.526 \mathrm{E}-003 & 1.160 \mathrm{E}-003 & 5.333 \mathrm{E}-004 & 4.375 \mathrm{E}-004 & 1.452 \mathrm{E}-003 & 1.387 \mathrm{E}-003 \\ 1.400 \mathrm{E}+001 & 2.586 \mathrm{E}-004 & 1.963 \mathrm{E}-004 & 9.183 \mathrm{E}-005 & 7.071 \mathrm{E}-005 & 2.538 \mathrm{E}-004 & 2.412 \mathrm{E}-004\end{array}$

\section{REFERENCE}

1. J. BRIESMEISTER (Editor), "MCNP-A General Monte Carlo N-Particle Transport Code, Version 4C," LA-13709-M, Los Alamos National Laboratory (2000). 